\title{
Necrotic Ulcerated Lesion in a Young Boy Caused by Cowpox Virus Infection
}

\author{
Anne-Laure Favier ${ }^{a} \quad$ Olivier Flusin $^{a} \quad$ Sébastien Lepreux ${ }^{b}$ \\ Hervé Fleury ${ }^{c}$ Christine Labrèze ${ }^{d} \quad$ Aurélie Georges $^{a}$ \\ Jean-Marc Crance ${ }^{a}$ Franck Boralevi ${ }^{d}$ \\ aUnité de Virologie, Institut de Recherche Biomédicale des Armées, IRBA, Antenne \\ de la Tronche-CRSSA, Grenoble, bService d'Anatomie Pathologique, Hôpital \\ Pellegrin, CHU Bordeaux, 'Laboratoire de Virologie, CHU de Bordeaux et UMR \\ 5234 CNRS, Université Victor Segalen, and dPediatric Dermatology Unit, Hôpital \\ Pellegrin-Enfants, Bordeaux, France
}

\section{Key Words}

Ulcerated lesion · Necrotic · Cowpox virus · Orthopoxvirus · Zoonosis · Rodent · Diagnosis - Hemagglutinin gene

\begin{abstract}
The case presented here points towards the fact that skin lesion observed with a cowpox virus is a rare event but should be considered more as the number of cases has increased in the last years. Cowpox virus (CPXV) belongs to the Poxviridae family. The transmission of CPXV to humans is caused by wild rodents or mostly by domestic animals and pet rats. In humans, CPXV is responsible for localized skin lesions regularly accompanied by lymphadenopathy. The lesions remain localized but self-inoculation from the primary lesions could occur. Then physicians have to be vigilant concerning bandages. In this case report, a necrotic and ulcerated lesion of a CPXV infection in a young boy is reported. The CPXV was possibly transmitted by wild rodents. The importance of performing the diagnosis is also pointed out. Virus information was obtained from phylogenetic analyses showing that the CPXV isolate was distinct from outbreaks of human cowpox which occurred in 2009 in France and Germany but was close to the CPXV Brighton Red strain. For several years, cases of viral zoonosis caused by CPXV have increased and physicians should be made aware that people could be infected without history of direct contact with animals.
\end{abstract}




\section{Introduction}

In recent years, several cowpox virus (CPXV) infections in humans have been described in Europe [1-6]. Cowpox virus, as well as monkeypox, vaccinia and variola virus, belong to the genus Orthopoxvirus, which can infect humans. Wild rodents are considered the reservoir host of CPXV and transmission to humans was shown to occur through infected animals, mostly domestic cats [7], but also pet rats [4, 5] and captive circus animals $[3,6]$. In immunocompetent humans, cowpox remains localized at the level of skin lesions which heal after several weeks; however, severe clinical cases have been described [2] and a fatal infection was reported in an immunocompromised patient [8]. Rodent-to-human transmission was reported after physical contact with animals or infection through skin abrasions, resulting in successive lesions for 2 weeks [7], but not often after a bite. We report a case of CPXV infection that occurred in a boy without documented contact with animals.

\section{Case Report and Methods}

A 10-year-old boy with no significant medical history, living in the southwest of France, presented with an initially small, red, infiltrated patch on the outside of his right arm which evolved quickly into a necrotic ulcer of $3 \mathrm{~cm}$ in size, surrounded by an erythematous infiltrated area (fig. 1A). The lesion was painless and the patient noted a low-grade fever of $38^{\circ} \mathrm{C}$ during the first days. Within several days, the erythema surrounding the ulcerated lesion extended to the entire arm, shoulder and axilla, associated with a morbilliform rash on his cheeks (fig. 1A). Physical examination showed regional solid axillar lymphadenopathy with no other systemic features. The patient was first treated with amoxicillineclavulinate for 5 days then with azithromycin $(10 \mathrm{mg} / \mathrm{kg} /$ day for 5 days $)$ with no improvement. Outcome was spontaneously favorable. The rash disappeared after 10 days, while lymph node enlargement persisted for 6 weeks and skin ulceration healed within 2 months by using hydrocolloid dressings.

Laboratory investigations were unremarkable except for a slight elevation of eosinophils ( $870 \mathrm{G} / \mathrm{l}$, $11 \%)$. Bacterial cultures were negative; no Leishmania parasite was identified on microscopic examination of skin scrapings. Serology for Coxiella burnetii, Rickettsia conorii, Borrelia burgdorferi, Rickettsia typhi, Rickettsia felis, and parvovirus B19 were negative. Assuming that the patient could suffer from tick-borne lymphadenopathy, detection of Rickettsia slovaca using PCR of skin biopsy was performed (D. Raoult, WHO Collaborative Center for Rickettsial Reference and Research, Marseille, France) and was negative. Light microscopic examination of a skin-punch biopsy showed full-thickness necrosis of the skin.

The patient had no history of skin injury in the affected region. He had no recent contact with animals such as rodents or cats, but he reported that he had played extensively in the hay on a farm near his home 12 days before the first lesions appeared. His parents reported that he never had direct contact with rodents, but the hay barn where he played regularly houses rodents.

\section{Diagnosis}

Electron microscopy examination of the isolate revealed orthopoxvirus-like particles (fig. 1B). A swab was sent to the French Armed Forces Biomedical Research Institute. Orthopoxvirus was diagnosed, after DNA extraction, in both the original swab and after culture in Vero cells by a $14-\mathrm{kDa}$ fusion protein real-time PCR [9]. Monkeypox virus-specific real-time PCR on both N3R and F3L genes [10] was negative. To identify the isolate (designated CPXV-F2010), the entire hemagglutinin (HA) gene was amplified [11]. The HA sequence (GenBank HQ634150) was compared to other orthopoxvirus and CPXV HA sequences available in GenBank and to CPXV HA sequences of clinical isolates including the viruses involved in the 2009 CPXV outbreaks in France and Germany $[4,5]$. 
A BLAST search performed for species identification of the reported case matched 8 published CPXV strains with an identity from 85 to $97 \%$ (www.ncbi.nlm.nih.gov/blast/Blast.cgi.). The HA sequences were submitted to a phylogenetic analysis (fig. 2A). The CPXV-F2010 strain clustered with 6 other cowpox virus strains available in GenBank including the Brighton Red strain (CPXV-BR). It differed from these CPXV strains between $3 \%$ to $7 \%$ at the nucleotide level. CPXV-F2010 is relatively close to CPXV-BR (97\% identity) and differs from outbreaks of human cowpox which occurred from December 2008 to January 2009 in France and Germany (CPXV-F2009 and CPXV-GER-rat2009, respectively). To improve the phylogenetic distribution of CPXV strains, 7 genes of the CPXV-F2010 and the CPXV-F2009 case were amplified (table 1) and sequenced to be concatenated [12].

Concatenated sequence alignment was performed and a phylogenetic tree was constructed (fig. 2B). This second phylogenetic tree confirmed the CPXV species of the reported CPXV-F2010 case. The sequences of 7 concatenated genes from CPXV-F2010 and CPXV-BR formed a clade, which is clearly distinct from other cowpox virus strains reported. The CPXV-F2010 strain differs from CPXV-BR by $2 \%$ at the nucleotide level.

Next, we investigated the in vitro host range, plaque phenotype and replication rate of CPXV-F2010 and CPXV-BR, the closely related strain identified by phylogenetic analysis (table 2). Four mammalian cell lines from various species and tissues were infected with the 2 viruses. CPXV-BR has a broad host range and multiplied in all the cell lines. CPXV-F2010 multiplied only in Vero, MRC5 and BHK-21 cells but not in mouse LA-4 cells. The lytic plaques obtained with CPXV-F2010 in Vero, VeroE6 and MRC5 cells were significantly smaller than those induced by CPXV-BR $(\mathrm{p}=0.0009, \mathrm{p}=0.0078$, and $\mathrm{p}=0.0146$, respectively; unpaired t-test). We could not determine the foci size on BHK-21 because of their imprecise outline morphology. Moreover, compared to CPXV-BR, CPXV-F2010 had significantly reduced virus multiplication in all the cell lines (data not shown). The data of in vitro phenotypic analyses suggest that CPXV-F2010 is different from CPXV-BR.

\section{Discussion}

We report CPXV infection in a 10 -year-old boy, with wild rodents as a possible source of infection. Although wild rodents are considered a natural reservoir of CPXV [13], only few reports have described wild rodent-to-human transmission $[1,14]$. The presence of wild rodents in the hay barn where the boy played 2 weeks before skin lesion appearance, suggests he might have been infected by contact with one of the rodents. Neither bites nor scratches having been felt by the patient, the mode of transmission is uncertain.

Epidemiologic investigations should be performed in the rodents living in this barn. The detection of CPXV in infected animals might clarify the origin of infection.

The diagnosis needs some specific molecular tools. The virus was identified as CPXV by EM, cell culture isolation, PCR and HA gene sequence analysis. Genotypic analyses on 7 concatenated genes showed that CPXV-F2010 formed a separate clade with cowpox virus strain CPXV-BR, isolated originally from a milker in the United Kingdom [15]. However, the phenotypic in vitro study showed that CPXV-F2010 differs from CPXV-BR, suggesting a new strain distinct from other CPXV strains reported so far.

This case highlights the risk for CPXV zoonoses in Europe where wild rodents are largely infected [13]. The risk of infection has increased since the cessation of smallpox vaccination and the consequent decrease of orthopoxvirus immunity. It also underscores the need for physicians and veterinarians to be aware of the risk for CPXV zoonoses and to investigate for cowpox in case of characteristic skin lesions in order to diminish the delay after several diagnostic and therapeutic detours. Although no cases of inter-human CPXV transmission have been reported, the risk of self-contamination has to be taken into account due to the scraping of the primary lesion by fingers. Semipermeable bandage should be used to cover the lesion site in order to provide significant protection from 
exposure to the virus on the outside of the bandage, and recommendations are necessary to avoid self-contamination with contaminated bandages.

\section{Acknowledgement}

We thank Samuel Forcet for excellent technical assistance.

\section{Financial Support}

This work was supported by research grants from the Service de Santé des Armées (SSA) and the Délégation Générale pour l'Armement (DGA).

\section{Disclosure Statement}

The authors declare that they have no conflict of interest.

Table 1. PCR primers used to sequence CPXV genes homologous to vaccinia virus, Copenhagen strain (VACV-COP)

\begin{tabular}{|c|c|c|c|}
\hline $\begin{array}{l}\text { VACV- } \\
\text { COP }\end{array}$ & Function & Forward primer & Reverse primer \\
\hline$A 56 R$ & Hemagglutinin & GCTGTCTTTCCTAAACCAG & GATAATGGTCACGTGTTACC \\
\hline$J 2 R$ & Thymidine kinase & GATACATAGATCCTCGTCGC & CGGTTTATCTAACGACACACAAC \\
\hline$D 4 R$ & Uracyl DNA glycosylase & CTTCATTTTGTCTGCACGC & GGCGTTTGTATTCGCTTG \\
\hline$A 48 R$ & Thymidilate kinase & CTTGGACAAGATGGACAGTC & CTGATTCCAAGTTGCCAGAG \\
\hline$B 5 R$ & Specific EEV protein, plaque size & CCACTGAAGAAGACATCTC & GTGCTCGACAGTGTATAC \\
\hline$C 22 L$ & Soluble TNF receptor & CTACCATCGGTCAATCTAG & GAGACGGTGTGAGAATATG \\
\hline$E 3 L$ & $\begin{array}{l}\text { Interferon resistance factor-apoptosis } \\
\text { inhibitor }\end{array}$ & CGCTCTACGAATATCTGTG & CGATAGGAACGACGAACCAC \\
\hline
\end{tabular}

PCR products were amplified with an annealing temperature of $52^{\circ} \mathrm{C}$ and using specific primers. 
Table 2. Plaque phenotype of CPXV-F2010

\begin{tabular}{|c|c|c|c|c|}
\hline Cell line & $\begin{array}{c}\text { CPXV-BR } \\
\text { (plaque size, } \mu \mathrm{m})\end{array}$ & Plaque aspect & $\begin{array}{c}\text { CPXV-F2010 } \\
\text { (plaque size, } \mu \mathrm{m} \text { ) }\end{array}$ & Plaque aspect \\
\hline VeroE6 & $5.53 \pm 1.22$ & & $4.15 \pm 1.26^{*}$ & \\
\hline Vero & $6.36 \pm 1.08$ & & $4.42 \pm 1.11^{*}$ & \\
\hline MRC5 & $15.78 \pm 3.40$ & & $9.88 \pm 4.38^{*}$ & \\
\hline BHK-21 & n.d. & & n.d. & \\
\hline LA-4 & $3.75 \pm 1.35$ & & No ECP & \\
\hline
\end{tabular}

Distribution of the mean foci size (mean \pm SD) of CPXV-BR (ATCC VR 302) compared to CPXVF2010 at $48 \mathrm{~h}$ p.i. in different cell lines. The mean foci size was determined by microscopy (Microscope Olympus CKX41) with an ocular micrometer after crystal violet staining of infected Vero, VeroE6, MRC-5 and LA-4 cells (ATCC CCL 81; ATCC CRL 1586; BioMérieux; ATCC CCL 196, respectively). Viral cytopathic effects were photographed with an Olympus C5050z camera.

* Significant differences: unpaired two-tailed $\mathrm{t}$ test; $\mathrm{p}<0.05$. 


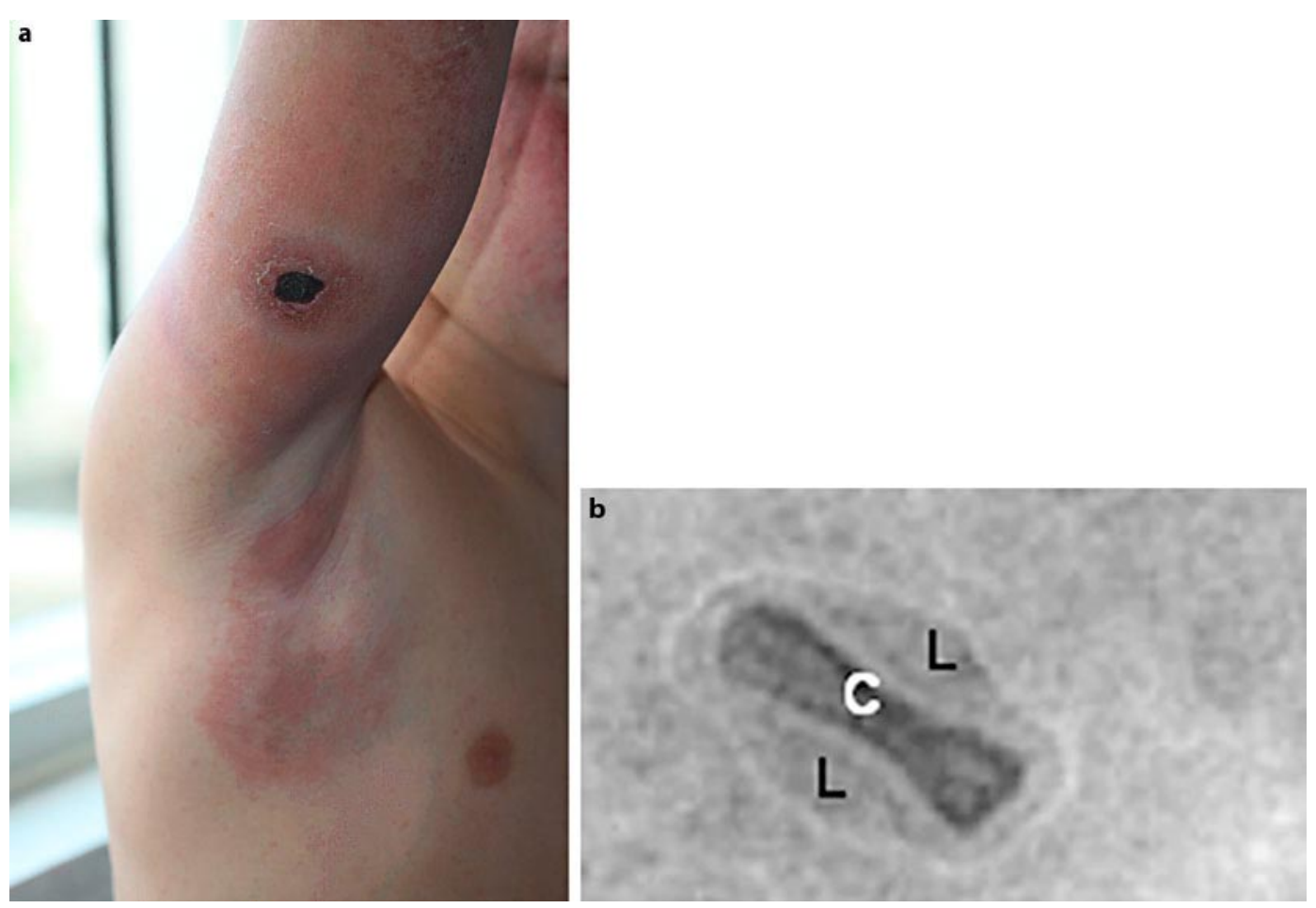

Fig. 1. Cowpox illustrations. a Lesion with necrotic ulcer on the outside of right arm with regional solid axillary lymphadenopathy. $\mathbf{b}$ Negative-staining electron microscopy of virus particle. $\mathrm{C}=\mathrm{Core} ; \mathrm{L}=$ lateral bodies. 
a

100 VARV-GAR



$\longmapsto 0.01$

b

b $\mathrm{CPXV}-\mathrm{F} 2010$

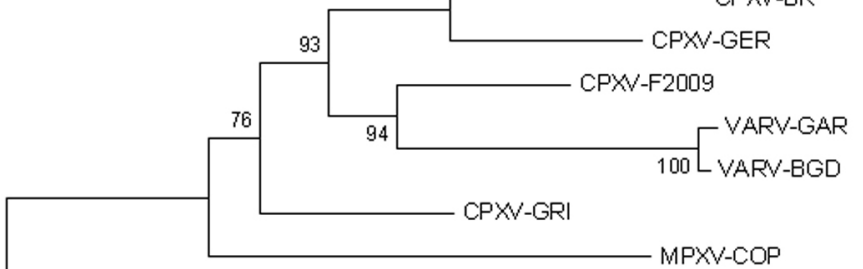

ECTV-MOS

0.01 
Fig. 2. Phylogenetic trees of selected orthopoxvirus genes. Sequence alignments were performed using the BioEdit program using BLOSUM62 similarity matrix. Phylogenetic analyses were performed from the BioEdit alignment with a global gap removal to exclude gaps from alignments. Neighbor-joining trees were constructed using the program MEGA3 with a Tamura-Nei substitution model. The robustness of trees was evaluated by bootstrap analysis with 1,000 rounds of replication. a Nucleotide sequences of the hemagglutinin gene from 2010-French human case (CPXV-F2010; HQ634150), 2009French human case (CPXV-F2009; FJ79031) and additional poxvirus available in GenBank: VARVBGD (variola major virus, strain Bangladesh; DQ441420), VARV-GAR (variola minor virus, strain Garcia; Y16780), MPXV-COP (monkeypox virus, strain Copenhagen; AY753185), CMLV-M96 (camelpox virus, strain M-96, AF438165), VACV-LIST (vaccinia virus, strain Lister-clone 107; DQ121394), ECTV-MOS (ectromelia virus, strain Moscow; AF012825), CPXV-BR (cowpox virus, strain Brighton Red; AF482758), CPXV-GRI (cowpox virus, strain GRI-90; X94355), CPXV-GER (cowpox virus, strain Germany 91-3; DQ437593), CPXV-FIN2003 (cowpox virus isolated from human in Finland in 2003, AY366478), CPXV-AUS2007 (cowpox virus isolated from human in Austria in 2007, EU582018), CPXV-cat1999 (cowpox virus isolated from cat in Germany in 1999, AF377885), CPXV-elephant2000 (cowpox virus isolated from elephant in Germany in 2000, AY902307). 2009 French rat-to-human outbreak (CPXV-FR-rat2009, bankit1198424), 2009 German rat-to-human outbreak (CPXV-GER-rat2009, FJ654467). The branch length is proportional to evolutionary distance (scale bar). b Phylogenetic tree of nucleotide sequences of the 7 concatenated genes. The 7 selected genes were concatenated to form a single sequence in the following order: $A 56 R, J 2 K, D 4 R, A 48 R, B 5 R$, $C 22 L$ and E3L. Nucleotide sequences from CPXV isolate from 2010 French human case (CPXV-F2010; HQ634150), 2009 French human case (CPXV-F2009; FJ79031) and additional poxvirus available in GenBank: VARV-BGD (variola major virus, strain Bangladesh; DQ441420), VARV-GAR (variola minor virus, strain Garcia; Y16780), MPXV-COP (monkeypox virus, strain Copenhagen; AY753185), VACV-LIST (vaccinia virus, strain Lister-clone 107; DQ121394), ECTV-MOS (ectromelia virus, strain Moscow; AF012825), CPXV-BR (cowpox virus, strain Brighton Red; AF482758), CPXV-GRI (cowpox virus, strain GRI-90; X94355), CPXV-GER (cowpox virus, strain Germany 91-3; DQ437593). The branch length is proportional to evolutionary distance (scale bar).

\section{References}

1 Wolfs TF, Wagenaar JA, Niesters HG, Osterhaus AD: Rat-to-human transmission of cowpox infection. Emerg Infect Dis 2002;8:1495-1496.

2 Pelkonen PM, Tarvainen K, Hynninen A, Kallio ER, Henttonen K, Palva A, Vaheri A, Vapalahti O: Cowpox with severe generalized eruption, Finland. Emerg Infect Dis 2003;9:1458-1461.

-3 Kurth A, Achenbach J, Miller L, Mackay IM, Pauli G, Nitsche A: Orthopoxvirus detection in environmental specimens during suspected bioterror attacks: inhibitory influences of common household products. Appl Environ Microbiol 2008;74:32-37.

4 Ninove L, Domart Y, Vervel C, Voinot C, Salez N, Raoult D, Meyer H, Capek I, Zandotti C, Charrel RN: Cowpox virus transmission from pet rats to humans, France. Emerg Infect Dis 2009;15:781-784.

5 Campe H, Zimmermann P, Glos K, Bayer M, Bergemann H, Dreweck C, Graf P, Weber BK, Meyer H, Buttner $\mathrm{M}$, Busch U, Sing A: Cowpox virus transmission from pet rats to humans, Germany. Emerg Infect Dis 2009;15:777-780.

6 Hemmer CJ, Littmann M, Lobermann M, Meyer H, Petschaelis A, Reisinger EC: Human cowpox virus infection acquired from a circus elephant in Germany. Int J Infect Dis 2010.

7 Baxby D, Bennett M, Getty B: Human cowpox 1969-1993: a review based on 54 cases. Br J Dermatol 1994;131:598-607.

-8 Eis-Hubinger AM, Gerritzen A, Schneweis KE, Pfeiff B, Pullmann H, Mayr A, Czerny CP: Fatal cowpox-like virus infection transmitted by cat. Lancet 1990;336:880.

-9 Scaramozzino N, Ferrier-Rembert A, Favier AL, Rothlisberger C, Richard S, Crance JM, Meyer H, Garin D: Real-time PCR to identify variola virus or other human pathogenic orthopox viruses. Clin Chem 2007;53:606613.

-10 Kulesh DA, Loveless BM, Norwood D, Garrison J, Whitehouse CA, Hartmann C, Mucker E, Miller D, Wasieloski LP Jr, Huggins J, Huhn G, Miser LL, Imig C, Martinez M, Larsen T, Rossi CA, Ludwig GV: Monkeypox virus detection in rodents using real-time 3 '-minor groove binder TaqMan assays on the Roche LightCycler. Lab Invest 2004;84:1200-1208. 
11 Ropp SL, Jin Q, Knight JC, Massung RF, Esposito JJ: PCR strategy for identification and differentiation of small pox and other orthopoxviruses. J Clin Microbiol 1995;33:2069-2076.

12 Garcel A, Crance JM, Drillien R, Garin D, Favier AL: Genomic sequence of a clonal isolate of the vaccinia virus lister strain employed for smallpox vaccination in France and its comparison to other orthopoxviruses. J Gen Virol 2007;88:1906-1916.

13 Chantrey J, Meyer H, Baxby D, Begon M, Bown KJ, Hazel SM, Jones T, Montgomery WI, Bennett M: Cowpox: Reservoir hosts and geographic range. Epidemiol Infect 1999;122:455-460.

14 Honlinger B, Huemer HP, Romani N, Czerny CP, Eisendle K, Hopfl R: Generalized cowpox infection probably transmitted from a rat. Br J Dermatol 2005; 153:451-453.

15 Fenner F, Burnet FM: A short description of the poxvirus group (vaccinia and related viruses). Virology 1957;4:305-314. 